\title{
Utilização de NBR Modificada com Grupos 2-0xazolina em Misturas de NBR/EVA
}

\author{
Mauricio S. M. Almeida, Bluma G. Soares \\ IMA, UFRJ \\ Pedro Ivo C. Guimarães \\ Instituto de Química, UERJ
}

\begin{abstract}
Resumo: Misturas envolvendo borracha nitrílica e copolímeros de etileno-acetato de vinila na proporção 70:30 \% em peso, foram preparadas na presença de borracha nitrílica funcionalizada com 2-oxazolina isoladamente ou combinada com EVA funcionalizado com grupos hidroxila, carboxila ou mercaptana. A reação entre os grupos funcionais foi verificada por análise de FTIR da fração insolúvel de amostras não vulcanizadas e pelo decréscimo nos valores de tan $\delta$ nos ensaios dinâmico-mecânicos. A utilização desses copolímeros funcionalizados não influenciou significativamente as propriedades mecânicas, provavelmente devido à proporção da mistura utilizada (rica em NBR) o que facilita a localização da NBR funcionalizada no interior da fase elastomérica.
\end{abstract}

Palavras-chave: Borracha nitrílica, 2-oxazolina, copolímeros de EVA, misturas poliméricas.

\section{Utilization of 2-0xazoline Modified NBR in NBR/EVA Blends}

Abstract : Nitrile rubber/ethylene-vinyl acetate copolymer (NBR/EVA) blends with ratio of 70/30 wt $\%$ were prepared in the presence of functionalized NBR with 2-oxazoline groups. This functionalized copolymer was employed alone or in combination with EVA copolymer functionalized with hydroxyl, carboxyl or mercaptan groups. The reaction between the functional groups was confirmed by FTIR analysis of the unvulcanized samples insoluble fraction, and by the decrease in the $\tan \delta$ values. The mechanical properties were not substantially affected by the presence of these functionalized copolymers, probably due to the proportion of blend composition (with high amount of NBR), thus forcing the location of functionalized NBR inside the elastomer phase.

Keywords: Nitrile rubber, 2-oxazoline, EVA copolymer, polymer blends.

\section{Introdução}

O campo da ciência e tecnologia de polímeros vem apresentando grande evolução durante as últimas décadas. Inicialmente, houve o desenvolvimento de novos polímeros a partir de uma variedade relativamente grande de monômeros. Em seguida, empregou-se a copolimerização estatística como uma técnica para produção de polímeros modificados. Foi desenvolvida então a copolimerização controlada em bloco e graftizada para a produção de novos materiais. Atualmente, há um grande interesse na área de misturas poliméricas, mesmo que as possibilidades de sínteses de polímeros ainda não tenham sido esgotadas. A mistura adequada de polímeros pode ser a melhor rota para a obtenção das propriedades desejadas, pois as misturas poliméricas, em geral, são implementadas mais rápido e economicamente, para atingir as demandas do mercado do que o desenvolvimento de novos polímeros ${ }^{[1]}$.

O uso de misturas não deve ser considerado estritamente como uma alternativa de competição para o desenvolvimento de novos polímeros, melhor que isso, ele pode oferecer um meio de se alcançar níveis de desempenho que não seriam viáveis através de um único polímero ${ }^{[2]}$.

Entretanto, muitas misturas apresentam incompatibilidade, resultando em sistemas pobres no que se refere às propriedades mecânicas. As propriedades de misturas incompatíveis são muito dependentes do tamanho e distribuição das fases (morfologia das misturas) e da adesão das interfaces ${ }^{[3-6]}$.

Um método que permite o controle desses aspectos é a modificação química da estrutura dos polímeros. Geralmente, essas reações são utilizadas para introduzir grupos funcionais reativos nos polímeros. Esses grupos reativos podem ser empregados como agentes compatibilizantes internos para misturas poliméricas ${ }^{[7,8]}$.

Entre os vários grupos reativos, a 2-oxazolina tem recebido destaque, uma vez que reagem com um grande número de outros grupos contendo hidrogênio lábil, tais como carboxilas, aminas, hidroxilas, fenóis e mercaptanas, tornando-se adequados para compatibilizar misturas com diversos polímeros ${ }^{[4,5,9-12]}$.

Materiais que estão sendo muito explorados ultimamente são os elastômeros termoplásticos, que associam a boa 
processabilidade dos termoplásticos com as propriedades elásticas das borrachas. Porém, se pode também, melhorar as propriedades de impacto de um termoplástico ao misturá-lo a pequenas quantidades de um elastômero ${ }^{[13]}$. Outra melhoria que pode ser obtida dessas misturas é o aumento da resistência ao envelhecimento, de uma borracha insaturada, pela adição de um termoplástico saturado como o copolímero de etileno e acetato de vinila (EVA) ${ }^{[2,14-16]}$.

O EVA apresenta excelentes resistência ao ozônio e a intempéries, com boas propriedades mecânicas a baixas temperaturas. O EVA já foi adicionado a várias borrachas, inclusive à borracha natural e ao policloropreno ${ }^{[17-20]}$. Encontram-se na literatura algumas citações sobre mistura com borracha nitrílica $(\mathrm{NBR})^{[13,18]}$. Essa mistura pode gerar uma importante classe de materiais com boa resistência a óleos, resistência à abrasão e boas propriedades mecânicas promovidas pela NBR, e resistência ao oxigênio e ozônio devido ao EVA. De acordo com a literatura essa mistura é imiscível e com propriedades insatisfatórias, em função da baixa adesão entre as fases. Esse problema pode ser minimizado pelo controle da mofologia durante o processamento ou pela adição de agentes compatibilizantes ${ }^{[13]}$.

O objetivo desse trabalho é avaliar vários sistemas de compatibilização reativa de misturas de borracha nitrílica com EVA. Utilizando, para tal fim, a incorporação de grupos reativos nos polímeros acima citados, através da modificação da NBR com grupos 2-oxazolinas e do copolímero de etileno e acetato de vinila com os grupos hidroxila, carboxila e mercaptana (Figura 1).

\section{Experimental}

\section{Materiais}

O NBR (33\% em massa de acrilonitrila; viscosidade Mooney $=50$ ) foi fornecido pela PETROFLEX. O copolímero EVA $(19 \%$ em massa de acetato de vinila; MFI $=2,5 \mathrm{~g} / 10$ min a $190^{\circ} \mathrm{C}$ ) foi cedido pela OPP Polietileno. O EVA modificado com grupos mercaptana (EVASH) foi obtido pela transesterificação do EVA com o ácido tioglicólico. A quantidade de grupos mercaptana incorporado ao polímero corresponde a $6,12 \%(\mathrm{~m} / \mathrm{m})$ em relação aos grupamentos acetato de vinila, como determinado por teor de enxofre ${ }^{[21]}$. O EVA parcialmente hidrolizado (EVALVA) foi produzido em tolueno pela reação com solução metanólica de hidróxido de sódio, obtendo-se um grau de hidrólise de 15,23 \%, como determinado por análise termogravimétrica (TGA) ${ }^{[21]}$. O EVA modificado com grupos carboxilas (EVALCOOH) foi obtido pela hidrólise total e posterior reação com anidrido maléico, resultando em incorporação de $18,00 \%$ de grupos carboxilas em relação ao acetato de vinila, segundo análises de TGA ${ }^{[21]}$. O NBR funcionalizado com grupos 2-oxazolina (NBROX) foi obtido conforme metodologia descrita por Hseih \& Peifer, obtendo-se modificação de $2,06 \%$ dos grupos nitrila em 2-oxazolina ${ }^{[22]}$. Óxido de zinco, ácido esteárico, enxofre, 2,2-ditiomercaptobenzotiazol (MBTS) e dissulfeto de tetrametiltiuram (TMTD) são todos de grau comercial.
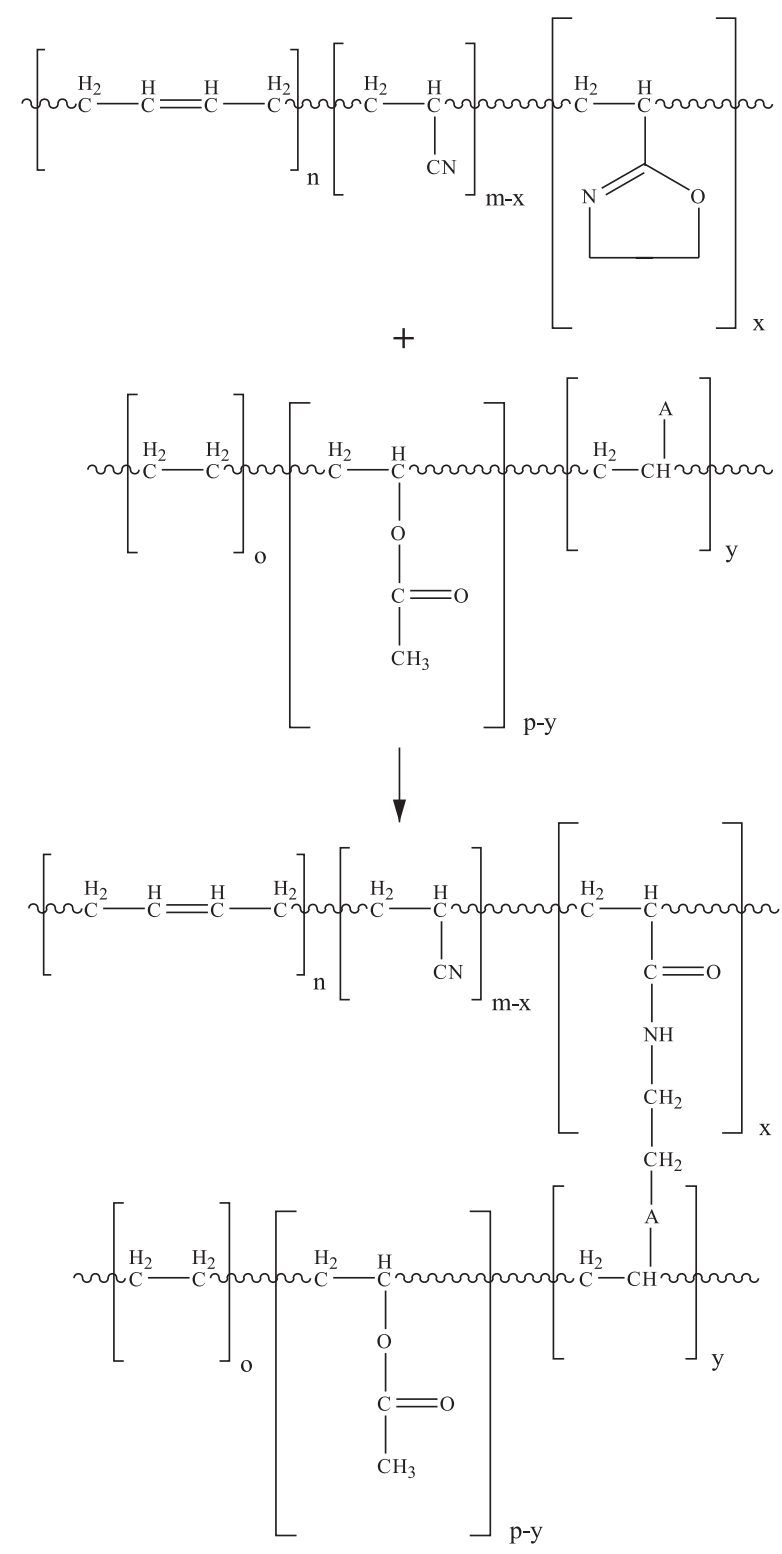

Figura 1. Esquema de compatibilização reativa entre NBR e EVA, onde A representa os grupos funcionais hidroxila, carboxila e mercaptana

\section{Viabilidade da Compatibilização}

Pequena quantidade de NBROX foi processada em um misturador de rolos Berstoff a $110^{\circ} \mathrm{C}$ e $25 \mathrm{rpm}$ por 3 minutos. Após esse tempo foi adicionado o EVA funcionalizado e misturado por mais 2 minutos. Foram preparadas três misturas na composição 50/50 de NBROX com EVALVA, EVALCOOH e EVASH, respectivamente. Para efeito de comparação realizouse também a mistura NBROX/EVA 50/50. Corpos de prova foram moldados por compressão em prensa hidráulica a $160{ }^{\circ} \mathrm{C}$ por 5 minutos. Esses corpos foram extraídos em tolueno a quente por 24 horas, e da fração insolúvel obteve-se espectros de FTIR (PERKIN-ELMER, Spectrum One).

\section{Preparação da Mistura}

As misturas NBR/EVA na composição 70/30 foram preparadas em um misturador de rolos Berstoff a $110^{\circ} \mathrm{C}$ e $25 \mathrm{rpm}$. 
Tabela 1. Composição das misturas NBR/EVA

\begin{tabular}{ccc}
\hline Ingredientes & $\begin{array}{c}\text { Quantidade } \\
\text { (phr) }\end{array}$ & $\begin{array}{c}\text { Tempo de mistura } \\
\text { (min) }\end{array}$ \\
\hline NBR & 70 & 3 \\
EVA & 30 & 2 \\
ZnO & 5,0 & 2 \\
Ácido esteárico & 0,5 & 2 \\
S & 0,30 & 2 \\
MBTS $*$ & 2,0 & 2 \\
TMTD** & 1,0 & \\
\hline
\end{tabular}

* 2,2-ditiomercaptobenzotiazol.

** dissulfeto de tetrametiltiuram.

O NBR foi mastigado por 2 minutos, em seguida adicionou-se 5 phr de NBROX, sendo misturado por mais 2 minutos, obtendo-se um "masterbatch" de NBROX. Os três "masterbatches" de EVA foram preparados da mesma forma. O EVA foi processado por 2 minutos. Adicionou-se então 5 phr de EVALVA, EVALCOOH e EVASH, respectivamente, permanecendo no rolo por mais 4 minutos. Foram preparadas 8 misturas 70/30 de NBR/EVA. A ordem de adição dos ingredientes da mistura, bem como quantidades e tempo de misturas são dados na Tabela 1 .

\section{Determinação dos Parâmetros Reométricos}

As misturas foram analisadas em reômetro de disco oscilatório (Tecnologia Industrial, mod. TI 100) a $160^{\circ} \mathrm{C}$ com arco $1^{\circ}$, de acordo com a norma ASTM D-2084-81.

\section{Propriedades Mecânicas}

Placas de $2 \mathrm{~mm}$ de espessura foram moldadas por compressão em uma prensa hidráulica a $160^{\circ} \mathrm{C}$, das quais corpos de prova do tipo halteres foram cortados. Esses corpos foram submetidos ao teste de tração em uma máquina de ensaio universal, Instron 4204, com velocidade de tracionamento de $200 \mathrm{~mm} / \mathrm{min}$ (DIN 53504).

\section{Análise Dinâmico-Mecânica}

Os ensaios de DMTA (Rheometric Scientific MKIII) foram realizados no modo de flexão de $-80{ }^{\circ} \mathrm{C}$ até $20^{\circ} \mathrm{C}$ com velocidade de aquecimento de $2^{\circ} \mathrm{C} / \mathrm{min}$ e frequência de $1 \mathrm{~Hz}$ para as misturas não vulcanizadas.

\section{Resultados e Discussão}

\section{Viabilidade da Compatibilização}

Com o intuito de se verificar a ocorrência de reação, determinou-se a fração insolúvel das misturas 50/50 apenas de polímeros funcionalizados. A Tabela 2 apresenta o teor de gel dessas misturas.

A mistura NBROX/EVA, como esperado, sofreu extração completa, uma vez que não existe grupo funcional que pudesse reagir com a 2-oxazolina. No caso das misturas reativas, isolou-
Tabela 2. Teor de gel de misturas 50/50 apenas de polímeros funcionalizados

\begin{tabular}{lc}
\hline \multicolumn{2}{c}{ Teor de gel } \\
\hline NBROX/EVA & $0 \%$ \\
NBROX/EVALVA & $25 \%$ \\
NBROX/EVALCOOH & $75 \%$ \\
NBROX/EVASH & $42 \%$ \\
\hline
\end{tabular}

se uma quantidade apreciável de material insolúvel, indicando a ocorrência de reação entre os grupos reativos. É curioso observar que a mistura compatibilizada com o par NBROX/EVALVA apresentou o menor teor de gel. Esse resultado indica que os grupos hidroxila não reagem tão eficientemente com os grupos oxazolina, como os grupos mercaptana ou carboxila. Por outro lado, a eficiência da compatibilização não está somente relacionada à eficiência da reação entre os grupos. Outros fatores, tais como viscosidade do sistema, devem ser levados em consideração. Neste sentido, é importante enfatizar que o copolímero EVALVA possui poucos grupos hidroxila ao longo da cadeia polimérica. A grande maioria dos grupos funcionais é ainda constituída de grupos acetoxi que confere uma viscosidade menor e, portanto, uma maior mobilidade no sistema.

Para comprovar a ocorrência da reação, foi realizado o espectro de infravermelho da fração insolúvel das amostras. Como a mistura NBROX/EVA foi totalmente extraída, utilizou-se um corpo de prova não extraído. O espectro dessa mistura fornece todas as bandas características da NBR e do EVA, além das bandas relativas às absorções do grupo 2 oxazolina, em $1664 \mathrm{~cm}^{-1}$ e $1260 \mathrm{~cm}^{-1}$, bandas que não aparecem nos demais espectros. Esse fato corrobora a ocorrência de reação entre os grupos reativos.

\section{Parâmetros Reométricos}

Os parâmetros de vulcanização obtidos a partir dos ensaios em reômetro de disco oscilatório encontram-se na Tabela 3. Nessa Tabela encontram-se também os valores de dureza e grau de inchamento, $\mathrm{Vr}$, uma vez que esses podem ser diretamente relacionados com o grau de ligações cruzadas na mistura.

Os valores de torque, bem como os de dureza e $\mathrm{Vr}$, sofreram apenas ligeiras variações, indicando que a densidade de ligações cruzadas não foi afetada de forma significativa com a adição dos polímeros funcionalizados. Quando copolímeros contendo grupos oxazolina ou mercaptana estavam presentes na mistura, o tempo de pré-cura, $\mathrm{t}_{\mathrm{s}} 1$, e o tempo ótimo de cura, ou seja, a $90 \%$ do torque máximo, $\mathrm{t}_{90}$, foram reduzidos. Esse fenômeno indica que tanto os grupos mercaptana como 2-oxazolina atuam como agentes auxiliares desse sistema de vulcanização $^{[17,20,23,24]}$.

\section{Propriedades Mecânicas}

$\mathrm{O}$ efeito da natureza do agente compatibilizante nas propriedades de tensão das misturas NBR/EVA é ilustrado nas Figuras 2 e 3 . Em todos os casos não houve grandes variações nas propriedades mecânicas com a adição desses polímeros. 
Tabela 3. Efeito dos copolímeros funcionalizados nos parâmetros reométricos, Vr e dureza das misturas NBR/EVA

\begin{tabular}{|c|c|c|c|c|c|c|c|c|}
\hline \multirow[b]{2}{*}{ Propriedades } & \multicolumn{8}{|c|}{ Misturas NBR/EVA contendo diferentes copolímeros funcionalizados } \\
\hline & - & NBROX & EVALVA & $\begin{array}{l}\text { NBROX } \\
\text { EVALVA }\end{array}$ & $\mathrm{EVALCO}_{2} \mathrm{H}$ & $\begin{array}{c}\text { NBROX } \\
\text { EVALCO }_{2} \mathrm{H}\end{array}$ & EVASH & $\begin{array}{l}\text { NBROX } \\
\text { EVASH }\end{array}$ \\
\hline $\mathrm{M}_{\mathrm{L}}(\mathrm{N} \cdot \mathrm{m})^{\mathrm{a}}$ & $0,19 \pm 0,01$ & $0,20 \pm 0,01$ & $0,18 \pm 0,01$ & $0,19 \pm 0,01$ & $0,18 \pm 0,01$ & $0,21 \pm 0,02$ & $0,19 \pm 0,01$ & $0,20 \pm 0,01$ \\
\hline $\mathrm{M}_{\mathrm{H}}(\mathrm{N} . \mathrm{m})^{\mathrm{b}}$ & $1,35 \pm 0,2$ & $1,23 \pm 0,2$ & $1,29 \pm 0,2$ & $1,20 \pm 0,1$ & $1,24 \pm 0,2$ & $1,31 \pm 0,1$ & $1,25 \pm 0,2$ & $1,28 \pm 0,2$ \\
\hline $\mathrm{M}_{\mathrm{H}}-\mathrm{M}_{\mathrm{L}}$ (N.m) & 1,16 & 1,03 & 1,11 & 1,01 & 1,06 & 1,10 & 1,06 & 1,08 \\
\hline $\mathrm{t}_{\mathrm{s}} 1(\min )^{\mathrm{c}}$ & $6,3 \pm 0,5$ & $4,5 \pm 0,3$ & $6,5 \pm 0,5$ & $3,9 \pm 0,5$ & $6,5 \pm 0,6$ & $4,5 \pm 0,5$ & $5,3 \pm 0,5$ & $3,7 \pm 0,5$ \\
\hline $\mathrm{t}_{90}(\min )^{\mathrm{d}}$ & $10,5 \pm 0,6$ & $8,9 \pm 0,2$ & $10,9 \pm 0,3$ & $8,2 \pm 0,5$ & $10,4 \pm 0,4$ & $9,2 \pm 0,5$ & $8,9 \pm 0,2$ & $8,3 \pm 0,6$ \\
\hline $\mathrm{Vr}^{\mathrm{e}}$ & $0,28 \pm 0,01$ & $0,29 \pm 0,01$ & $0,28 \pm 0,01$ & $0,29 \pm 0,01$ & $0,29 \pm 0,01$ & $0,30 \pm 0,01$ & $0,28 \pm 0,01$ & $0,29 \pm 0,01$ \\
\hline Dureza (ShoreA) & $62 \pm 1$ & $61 \pm 1$ & $62 \pm 1$ & $63 \pm 1$ & $63 \pm 1$ & $63 \pm 1$ & $62 \pm 1$ & $62 \pm 1$ \\
\hline
\end{tabular}

${ }^{a}$ torque mínimo $\quad{ }^{\mathrm{b}}$ torque máximo $\quad{ }^{\mathrm{c}}$ tempo de pré-cura $\quad{ }^{\mathrm{d}}$ tempo a $90 \%$ do torque máximo ${ }^{\mathrm{e}}$ grau de inchamento

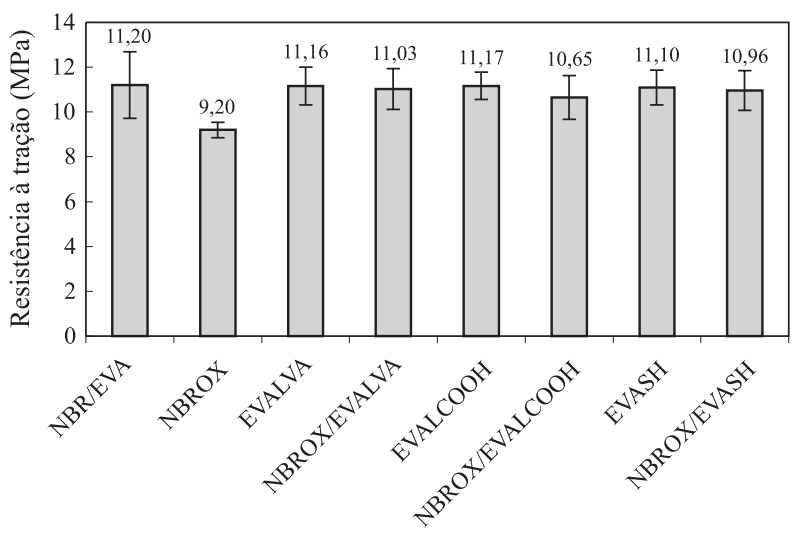

Figura 2. Efeito da natureza do agente de compatibilização na resistência à tração de misturas NBR/EVA

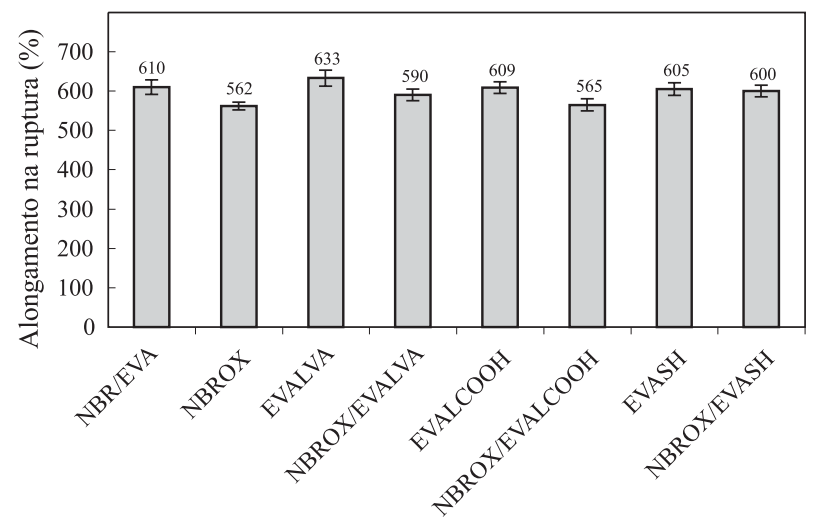

Figura 3. Efeito da natureza do agente de compatibilização no alongamento na ruptura de misturas NBR/EVA

Apesar da conhecida reatividade dos grupos 2-oxazolina, a presença desses nas misturas não ocasionou melhora alguma nas propriedades. Quando a NBROX foi empregada isoladamente, ocorreu uma ligeira redução no valor da resistência à tração.

Como já foi discutido, os grupos 2-oxazolina são capazes de reagir com os demais grupos funcionais localizados na fase EVA. Entretanto, o copolímero graftizado (reticulado) resultante pode estar sendo encapsulado por uma das fases da mistura, perdendo assim a sua ação interfacial.

No caso da mistura contendo apenas NBROX como agente compatibilizante, o decréscimo da resistência à tração pode ser devido à sua possível reação com o ácido esteárico, componente do sistema de vulcanização, o que prejudica a formação de ligações cruzadas. Este efeito é, entretanto, muito pequeno para alterar os valores de $\mathrm{Vr}$.

$\mathrm{O}$ alongamento na ruptura sofreu apenas ligeiras variações, não alterando o comportamento das misturas de forma significativa.

\section{Análise Dinâmico-Mecânica}

A dependência do fator de amortecimento $(\tan \delta)$ com a temperatura para misturas compatibilizadas com o sistema a base de EVALVA é apresentada na Figura 4. Embora as misturas sejam incompatíveis, a transição vítrea das fases NBR e EVA ocorre a uma temperatura bem próxima, dando origem a apenas um sinal ${ }^{[13]}$. Verifica-se uma discreta redução do fator de amortecimento, $\tan \delta$, para as misturas

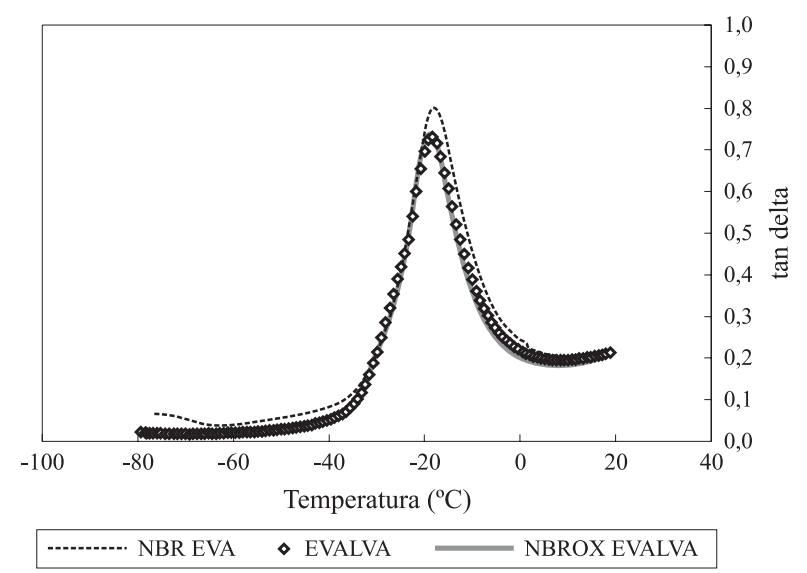

Figura 4. Curva do fator de amortecimento das misturas não vulcanizadas contendo EVALVA 


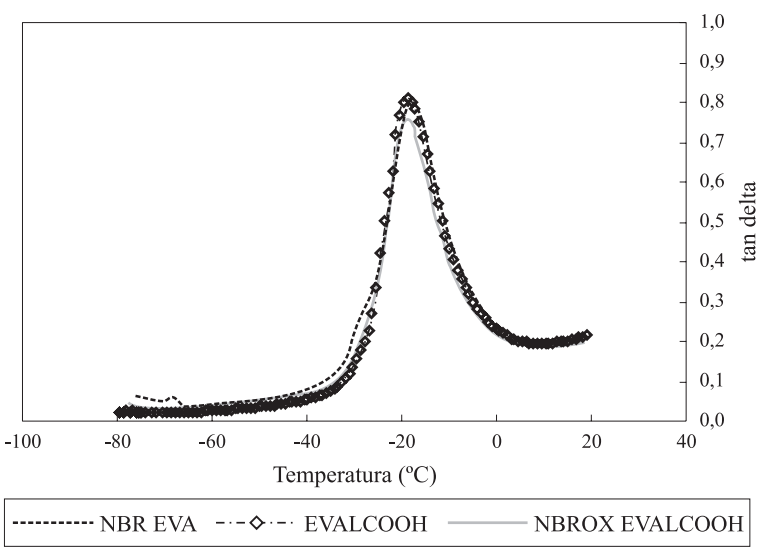

Figura 5. Curva do fator de amortecimento das misturas não vulcanizadas contendo EVALCOOH

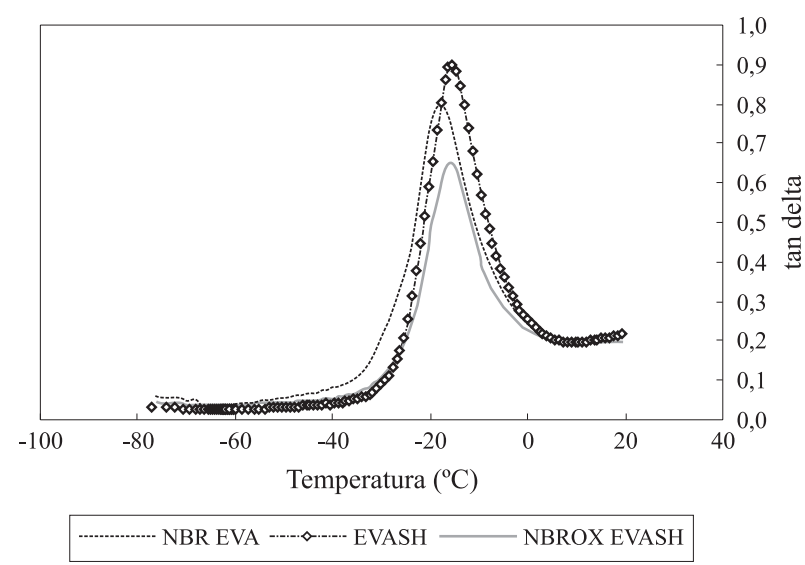

Figura 6. Curva do fator de amortecimento das misturas não vulcanizadas contendo EVASH

compatibilizadas com EVALVA isoladamente ou NBROX/ EVALVA. Essa tendência sugere uma pequena redução da flexibilidade das cadeias poliméricas devido às prováveis interações físicas ou químicas entre as fases. No caso da compatibilização com EVALVA, acredita-se que interações do tipo ligações hidrogênio estejam contribuindo para uma discreta diminuição da flexibilidade das cadeias.

A Figura 5 compara o comportamento da $\tan \delta$ de misturas não compatibilizadas com aquelas compatibilizadas com EVALCOOH e NBROX/EVALCOOH. Apesar do maior número de grupos polares na amostra EVALCOOH em relação aos demais copolímeros funcionalizados, utilizados nesse trabalho, a dependência da $\tan \delta$ com a temperatura é similar àquela da mistura não compatibilizada. Essa diferença entre os comportamentos das misturas compatibilizadas com EVALVA e com EVALCOOH isoladamente pode estar associada a uma menor viscosidade da amostra EVALVA, o que contribui para uma melhor difusão da amostra no sistema, facilitando as interações entre as fases. A mistura contendo o par NBROX/EVALCOOH apresentou ligeira queda no amortecimento, sugerindo a ocorrência de reação entre os grupos 2-oxazolina e carboxila.

A mistura contendo NBROX/EVASH (Figura 6) sofreu uma queda mais acentuada no fator de amortecimento. Este decréscimo indica decréscimo na mobilidade das cadeias, provavelmente devido à ocorrência de reações durante o processamento. Os resultados dinâmico-mecânicos obtidos com a utilização de pares reativos em misturas NBR/EVA sugerem que a reatividade dos grupos oxazolina com os grupos SH é maior do que com os grupos carboxila.

\section{Conclusões}

A adição de polímeros modificados reduziu o tempo de pré-cura, bem como o tempo ótimo de vulcanização, mas não alterou significativamente os torques máximos e mínimos das misturas.

As propriedades mecânicas não foram influenciadas pela adição dos copolímeros funcionalizados, exceto no caso da NBROX, quando utilizada isoladamente, que apresentou um decréscimo nos valores de tensão na ruptura. Acredita-se que a mistura contendo alto teor de NBR favoreça a localização da NBROX dentro da fase elastomérica, diminuindo assim o contato na interface. No caso da utilização da NBROX isoladamente, a adição do ácido esteárico pode estar contribuindo para uma desativação do poder reativo da NBROX. Além disso, a própria reação entre NBROX e o ácido esteárico utilizado como ativador no processo de vulcanização esteja diminuindo a sua concentração, prejudicando as propriedades finais do material.

\section{Agradecimentos}

Os autores agradecem à CAPES, CEPG-UFRJ, CNPq e PADCT/CNPq (Proc. $\mathrm{n}^{\circ}$ 620132/98-1) pelo apoio financeiro e à PETROFLEX S.A. e à OPP POLIETILENO S.A. pela doação dos polímeros.

\section{Referências Bibliográficas}

1. Paul, D. R.; Barlow, J. W. \& Keskkula, H. - "Polymer blends". In "Encyclopedia of Polymer Science and Technology", editado 'por Mark, H.M., Bikales, N.M., Overberg, C.G., Menges, G., John Wiley \& Sons, New York, volume 12, pp. 399 (1986).

2. Koning, C.; Duin, M. V. ; Pagnoulle, C. \& Jerome, R. "Strategies for compatibilization of polymer blends", Prog. Polym. Sci. 23, 707-757 (1998).

3. Takeda, Y. \& Paul, D. R. - "Morphology of Nylon-6 blends with styrenic polymers", J. Polym. Sci.: Part B: Polym. Phys. 30, 1273-1284 (1992).

4. Tan, N. C. B.; Tai, S.-K. \& Briber, R. M. - "Morphology control and interfacial reinforcement in reactive polystyrene/amorphous polyamide blends", Polym. 37 (16), 3509-3519 (1996).

5. Hietaoja, P.; Heino, M.; Vainio, T. \& Seppälä, J. "Compatibilization of PP/PBT and PP/PA6 blends with a new oxazoline-functionalized polypropylene", Polym. Bull. 37, 353-359 (1996). 
6. Liu, N. C. \& Baker, W. E. - "Basic functionalization of polypropylene and the role of interfacial chemical bonding in its toughening", Polym. 35 (5), 988-994 (1994).

7. Liu, N. C. \& Baker, W. E. \& Xie, H. Q. - "Comparison of the effectiveness of different basic functional groups for the reactive compatibilization of polymer blends", Polym. 34 (22), 4680-4687 (1993).

8. Rösch, J.; Warth, H.; Müller, P.; Schäfer, R.; Wörner, C.; Friedrich, C.; Kressler, J. \& Mülhaupt, R. - "Prospects and challenges of reactive processing", Macromol. Symp. 102, 241-254 (1996).

9. Anttila, U.; Vocke, C. \& Seppälä, J. - "Functionalization of Polyolefins and elastomers with an oxazoline compound”, J. Appl. Polym. Sci. 72, 877-885 (1999).

10. Hu, G.-H.; Scaffaro, R. \& Mantia, F. - "Chemical modificatio of nitrile oxazoline functionality on a stytene-acrylonitrile copolymer in melt", J. M. S. - Pure Appl. Chem. A35 (3), 457-471 (1998).

11. Vainio, T.; Hu, G.-H.; Lambla, M. \& Seppälä, J. "Functionalization of polypropylene with oxazoline and reactive blending of $\mathrm{PP}$ with $\mathrm{PBT}$ in a corotating twin-screw extruder", J. Appl. Polym. Sci. 63 (7), 883894 (1997).

12. Vainio, T.; Hu, G.-H.; Lambla, M. \& Seppälä, J. V. "Functionalized polypropylene prepared by melt free radical grafting of low volatile oxazoline and its potential in compatibilization of PP/PBT blends", J. Appl. Polym. Sci. 61, 843-852 (1996).

13. Soares, B. G. \& Jansen, P. - "Mercapto-modified copolymers in polymers blends. II. The compatibilization of NBR/EVA blends", J. Appl. Polym. Sci. 79, 193-202 (2000)

14. Aoi, K. \& Okada, M. - "Polymerization of oxazolines", Prog. Polym. Sci. 21, 151-208 (1996).
15. Paul, D. R.; Barlow, J. W. \& Keskkula, H. - "Polymer blends". In "Encyclopedia of Polymer Science and Technology", editado 'por Mark, H. M., Bikales, N. M., Overberg, C. G., Menges, G., John Wiley \& Sons, New York, volume 12, pp. 399 (1986).

16. Gesner, B. D. - "Polyblends". In "Encyclopedia of Polymer Science and Technology", editado 'por Mark, H.M., Bikales, N.M., Overberg, C.G., Menges, G., John Wiley \& Sons, New York, volume 10, pp. 694 (1985).

17. Aoi, K. \& Okada, M. - "Polymerization of oxazolines", Prog. Polym. Sci. 21, 151-208 (1996).

18. Allen, C. F. H. \& Dunlop, A. P. - "Heterocyclic compounds". In "Encyclopedia of Chemical Technology", editado por A. Standen, John Wiley \& Sons Inc., New York, volume 10, second edition, pp. 895-903 (1966).

19. Meyers, A. I. \& Mihelich, E. D. - "The synthetic utility of 2-oxazolines", Angew. Chem. Int. Ed. Engl. 15 (5), 270-281 (1976).

20. Wiley, R. H. \& Bennett Jr., L. L. - "The chemistry of oxazolines", Chem. Reviews 44, 447-476 (1949).

21. Dutra, R. C. L.; Lourenço, V. L.; Diniz, M. F.; Azevedo, M. F. P.; Barbosa, R. V. \& Soares, B. G. - Polym. Bull. 36, 593 (1996).

22. Hseih, D. T. \& Peiffer, D. G. - "Chemical transformation of nitrile to reactive oxazoline functionality on a preformed polymer", J. Appl. Polym. Sci. 56, 1667-1671 (1995).

23. Frump, J. A. - "Oxazolines. Their preparation, reactions, and applications", Chem. Reviews 71 (5), 483-505 (1971).

24. Kobayashi, S. - "Ethylenimine polymers", Prog. Polym. Sci. 15, 751-823 (1990).

Recebido: $10 / 08 / 01$

Aprovado: 21/02/03 